\title{
Identification of impact force for smart composite stiffened panels
}

\author{
M. Ghajari ${ }^{1}$, Z. Sharif-Khodaei ${ }^{1}$, M.H. Aliabadi ${ }^{1}$ and A. Apicella ${ }^{2}$ \\ ${ }^{I}$ Department of Aeronautics, Imperial College London, South Kensington Campus, \\ SW7 2AZ, London, UK \\ ${ }^{2}$ Alenia, Viale dell'Aeronautica, Pomigliano d'arco, Italy
}

\begin{abstract}
In this work, a new methodology is presented for reconstructing the impact force history using Artificial Neural Networks (ANNs) and spectral components of sensor data recorded by piezoceramic sensors. A large set of data, required for training the ANNs, were generated by using an efficient nonlinear Finite Element (FE) model of a sensorised composite stiffened panel. Impact experiments were performed on a composite plate equipped with surface-mounted piezoceramic sensors to validate the numerical modelling approach. Using the FE model of the panel, data were generated for impacts which are likely to occure during life-time of an aircraft, containing large mass (e.g. dropping tool) and small mass (e.g. debris) impacts at various locations, i.e. in bay, on the foot of stringer and over/under stringer. Even though the panel undergoes large deformation during impact (nonlinear response), the established networks predict the impact force history and its peak with reasonable accuracy.
\end{abstract}

Keywords: impact, force reconstruction, structural health monitoring, artificial neural network, composite stiffened panel

\section{Introduction}

In the aviation industry, real-time characterisation of impact events has direct influence on design, production and maintenace costs. The impact characterisation contains estimating the location and magnitude of the impact by using the data collected via sensors. This is an inverse problem; the input to the system is estimated from its response. In practice, the response of the structure subjected to an impact is recorded via embedded or surface-mounted sensors. These data are subsequently analysed with a suitable algorithm to locate the impact and determine the peak impact force or possibly reconstrcut the impact force history. Determination of the impact location on composite stiffened panels has been addressed in several studies (Dae-Un et al. 2000; Worden et al. 2000; Haywood et al. 2005; Park et al. 2009; SharifKhodaei et al. 2012). The focus of this paper is on determination of the impact force.

In the last three decades, several methods have been proposed to reconstruct the impact force from the response of the system by taking advantage of the convolution integral. A detailed review of some of these methods can be found in (Inoue et al. 2001). In (Doyle 1987; Inoue et al. 1991; Martin et al. 1996), the frequency domain deconvolution method was employed to estimate spectral components of the impact force, and then the time history of the force was obtained by using the inverse fast Fourier transform. Doyle (1997) used wavelet functions in conjunction with the convolution integral to develop a new algorithm for estimating the impact force history. This method provided more accurate results than the frequency domain deconvolution method. Park et al. (2009) proposed a method that is applicable to complex structures. In this method, the relationship between the impact force and the sensor data was represented by a linear finite difference model, and the unkowns of 
the model were determined by using a set of training data, which were obtained experimentally. This method was also based on the convolution integral. Identification of multi-site impacts, based on using the convolution relation, has been addressed in (Adams et al. 2002; Jankowski 2009).

Artifitial Neural Networks (ANNs) have also been used to predict the peak impact force using different features of the sensor signal (Jones et al. 1997; Staszewski et al. 2000; Worden et al. 2000; Haywood et al. 2005). ANNs are mathematical models that can be trained to model complex nonlinear relationships between the inputs and outputs. Once trained, they provide real-time predictions, similar to other pattern recognition techniques, which can be crucial for some applications such as adaptive impact absorption systems (Sekuła et al. 2013). In (Staszewski et al. 2000), 80 impacts were carried out on a composite panel at random locations in order to provide data for training. The level of the force was kept below $0.1 \mathrm{~N}$ in order not to damage the plate. For the ANN with the best performance, the mean error of the estimated peak force on the test data was $28 \%$. In a previous study by the authors (Ghajari et al. 2012), an ANN was established and trained for detecting impact events on various locations of an aircraft composite stiffened panel. In contrast to the majority of previous studies, both small mass and large mass impacts were considered. The mean error of the peak force measured on the test data was slightly better than previous studies, approximately $26 \%$.

The force reconstruction methods based on the convolution integral are restricted to linear cases, which implies that impact forces should be so small that they do not cause large deflections (geometric nonlinearity) in the structure. However, aircraft panels are very likely to undergo large deflections during life-time impacts, such as dropping tool, debris impact or hail strike. In addition, there are no other efficient methods that can be applied to real-time reconstruction of the impact force when there is geometric nonlinearity.

To overcome this problem, in the present paper, a new methodology based on Artificial Neural Networks is presented to reconstruct the force history of impacts which cause large deflections in the panel. A large set of data, required for training the networks, were generated by using an efficient nonlinear Finite Element (FE) model of a composite stiffened panel. Different types of impacts that occur during life-time of an aircraft were simulated. The impacts were applied at various locations (in bay, on the foot of stringer, over/under stringer) and from inside and outside of the panel. ANNs were established to reconstruct the force and to estimate the peak force. The effects of signal features, network architecture, noise and sensor distribution on the performance of the ANNs are investigated. Signal processing techniques are employed to reveal possible differences between sensor signals recorded in small mass and large mass impacts, which can be used to categorise impacts.

The paper is organised as follows: in section 2, the frequency domain deconvolution method is explained. Results of impact experiments on a sensorised composite plate and validation of the modelling approach are presented in section 3 . In the same section, the applicability of the frequency domain deconvolution method to impact force reconstruction of thin plates is examined. Section 4 is dedicated to describing the proposed ANN based methodology for force reconstruction and determination of the peak force. Some discussions are presented in section 5 following by concluding remarks in section 6 . 


\section{Force reconstruction using frequency domain deconvolution}

An aircraft stiffened panel is typically composed of a skin, stiffeners and stringers. During an impact, part of impactor's kinetic energy converts to strain waves propagating in the skin from the impact location. These waves, which can be measured with surface bonded piezoceramic sensors, contain some information about the impact event. For instance, the time that it takes for the strain wave to reach a sensor (time of arrival, ToA) has a direct relation with the distance between the impact location and the sensor. Analysis of ToAs of a few sensors with the triangulation method or ANNs (Dae-Un et al. 2000; Sharif-Khodaei et al. 2012) leads to precise determination of the impact location.

Sensor signals also contain some information about the impact force. To reveal these information, it may be assumed that the dynamical system (s) is linear timeinvariant (LTI), which means for any two input signals $f_{l}(t)$ and $f_{2}(t)$ and any real constant $a$ :

$$
\begin{gathered}
s\left[f_{1}(t)+f_{2}(t)\right]=s\left[f_{1}(t)\right]+s\left[f_{2}(t)\right] \\
s\left[a f_{1}(t)\right]=a s\left[f_{1}(t)\right]
\end{gathered}
$$

and if $\mathrm{u}_{1}(\mathrm{t})=\mathrm{s}\left[\mathrm{f}_{1}(\mathrm{t})\right]$ then $\mathrm{u}_{1}(\mathrm{t}-\mathrm{T})=\mathrm{s}\left[\mathrm{f}_{1}(\mathrm{t}-\mathrm{T})\right]$, where $\mathrm{t}$ and $\mathrm{T}$ are time. The latter condition is normally satisfied for composite stiffened panels subjected to impacts, as there is no significant time-dependent variable, such as a rate-sensitive material, in the system. The former conditions are satisfied when deflection of the panel under impact loading is not large. As a rule of thumb, deflection of thin-walled structures is large when it exceeds the thickness of the plate (Abrate 2001). This condition will be discussed later.

Essentially, all LTI systems can be represented with a time convolution integral. Assuming zero initial conditions, $\mathrm{u}(0)=0$, this integral is:

$$
\mathrm{u}(\mathrm{t})=\int_{0}^{\mathrm{t}} s(\mathrm{t}-\tau) \mathrm{f}(\tau) \mathrm{d} \tau
$$

The input, $f(t)$, can be the impact force history and the response, $u(t)$, can be the inplane strain measured with a sensor. In the frequency domain, the convolution equation becomes:

$$
U\left(\omega_{n}\right)=S\left(\omega_{n}\right) F\left(\omega_{n}\right)
$$

where:

$$
\begin{aligned}
& f\left(t_{k}\right)=\frac{1}{N} \sum_{n=1}^{N} F\left(\omega_{n}\right) e^{i \omega_{n} t_{k}} \\
& u\left(t_{k}\right)=\frac{1}{N} \sum_{n=1}^{N} U\left(\omega_{n}\right) e^{i \omega_{n} t_{k}} \\
& s\left(t_{k}\right)=\frac{1}{N} \sum_{n=1}^{N} S\left(\omega_{n}\right) e^{i \omega_{n} t_{k}}
\end{aligned}
$$

The above equations are discrete spectral representations of the force, sensor signal and system. Capital letters are used to indicate the frequency domain representation of quantities. The circular frequency is: 


$$
\omega_{n}=\frac{2 \pi(n-1)}{N \Delta t}
$$

where $\mathrm{N}$ is the total number of data points and $\Delta \mathrm{t}$ is the sampling time-step. Equation (3) suggests a relatively simple solution to the force reconstruction problem; given frequency domain representations of the system and the sensor signal, the components of the spectral force can be obtained from:

$$
F\left(\omega_{n}\right)=U\left(\omega_{n}\right) / S\left(\omega_{n}\right)
$$

If $\mathrm{S}\left(\omega_{\mathrm{n}}\right)$ is zero, the inverse problem becomes ill-conditioned. Martin and Doyle (1996) have proposed a remedy for this problem, which will be discussed in section 3.2. An inverse Fourier transform of the spectral force will provide the reconstructed force in the time domain. $S$ depends on the material properties and geometry of the problem. For simple problems, it can be determined by using analytical solutions (Doyle 1987). However, for complex problems, experiments or numerical simulations should be performed to accurately determine $S\left(\omega_{\mathrm{n}}\right)$ (Inoue et al. 1991; Gopalakrishnan et al. 1994).

\section{Force reconstruction for impacts on a composite plate}

The frequency domain deconvolution method was used to reconstruct the force history of impacts on a composite plate. The impact events and wave propagation were simulated with the Finite Element (FE) method. An advantage of using the FE method is that the validity of assumptions made for force reconstruction, particularly the linearity of the system, can be easily examined. To validate the accuracy of the FE model, impact tests were carried out on the sensorised composite plate and the results were compared to numerical predictions.

\subsection{Validation of the FE model}

The plate was made of HTA/6376C carbon fibre reinforced epoxy resin, with a $\left[0 /+45 /-45 / 90 / 0_{2} / 90 / 0 /+45 /-45 / 0 / 90\right]_{S}$ lay-up and a $3.4 \mathrm{~mm}$ thickness. This laminate has applications in skin-stringer aircraft panels. Two circular piezoceramic sensors (PIC 255), $10 \mathrm{~mm}$ diameter and $0.5 \mathrm{~mm}$ thickness with wrap-around electrodes, were bonded on the plate with an epoxy resin (Araldite 2011). Material properties of the composite lamina and the sensor are presented in Table 1.

\begin{tabular}{|c|c|c|c|c|c|c|}
\hline \multirow{2}{*}{ HTA/6376C } & $\mathrm{E}_{1}[\mathrm{GPa}]$ & $\mathrm{E}_{2}[\mathrm{GPa}]$ & $v_{12}$ & $\mathrm{G}_{12}=\mathrm{G}_{13}[\mathrm{GPa}]$ & $\mathrm{G}_{23}[\mathrm{GPa}]$ & $\rho\left[\mathrm{kg} / \mathrm{m}^{3}\right]$ \\
\hline & 137 & 9.9 & 0.3 & 5.2 & 3.1 & 1300 \\
\hline \multirow{2}{*}{ PIC 255} & $\mathrm{E}$ [GPa] & $\mathrm{v}$ & $\rho\left[\mathrm{kg} / \mathrm{m}^{3}\right]$ & & & \\
\hline & 100 & 0.38 & 7800 & & & \\
\hline
\end{tabular}

Table 1 Material properties of the composite lamina and sensor

The plate was supported in a fixture with a $200 \mathrm{~mm}$ by $200 \mathrm{~mm}$ window, representing a typical stringer pitch in aircaft panels. Impacts were carried out using an Instron impact system. The impactor had a $10 \mathrm{~mm}$ radius tup and its mass was 2.41 $\mathrm{kg}$. The impactor was dropped at the centre of the plate from a height of $43 \mathrm{~mm}(0.92$ $\mathrm{m} / \mathrm{s}$ initial velocity). Impact energy was kept low in order to avoid damaging the plate. 
The impact force and sensor data were recorded at a frequency of $200 \mathrm{kHz}$. The experiment was repeated three times. Very good repeatability of the results was observed, which indicates that the plate was not damaged during the impacts.

The impact test was simulated, using the ABAQUS FE software (2010). The plate was meshed with $2 \mathrm{~mm}$ general purpose shell elements (S4R) and the compsite lay-up was defined with through-thickness integration points. Following suggestions made by (Sharif-Khodaei et al. 2012), instead of modelling the small sensor with solid elements, which drastically decreases the time step and consequently increases the simulation time, one through-thickness integration point was added to the shell elements at the location of the sensor to represent the sensor. Figure 1 shows the dimensions of the plate, location of the sensors and boundary conditions. The impactor was modelled with discrete-rigid elements. The softened penalty algorithm, with the pressure-overclosure formulation, was used to model the contact between the plate and the impactor. The pressure-overclosure data were determined by using the following relation, which was derived based on the Hertzian contact theory in (SharifKhodaei et al. 2012):

$$
p=\frac{3 k_{H} y^{1 / 2}}{4 \pi R}
$$

In this equation, $\mathrm{p}$ is the contact pressure, $\mathrm{y}$ is overclosure of slave nodes (nodes of the impactor), $\mathrm{R}$ is the radius of the impactor and $\mathrm{k}_{\mathrm{H}}$ is the contact stiffness, which is dependent on the mechanical properties of the impactor and the laminate. Contact stiffness was determined as $6.6 \times 10^{8} \mathrm{Nm}^{3 / 2}$ by using the relations presented in (Olsson 2001) and scaling the resulting value with respect to the theoretical and experimental values of $\mathrm{k}_{\mathrm{H}}$ obtained for the contact between a steel sphere and a carbon-fibre reinforced polymer laminate (Pierson et al. 1996).

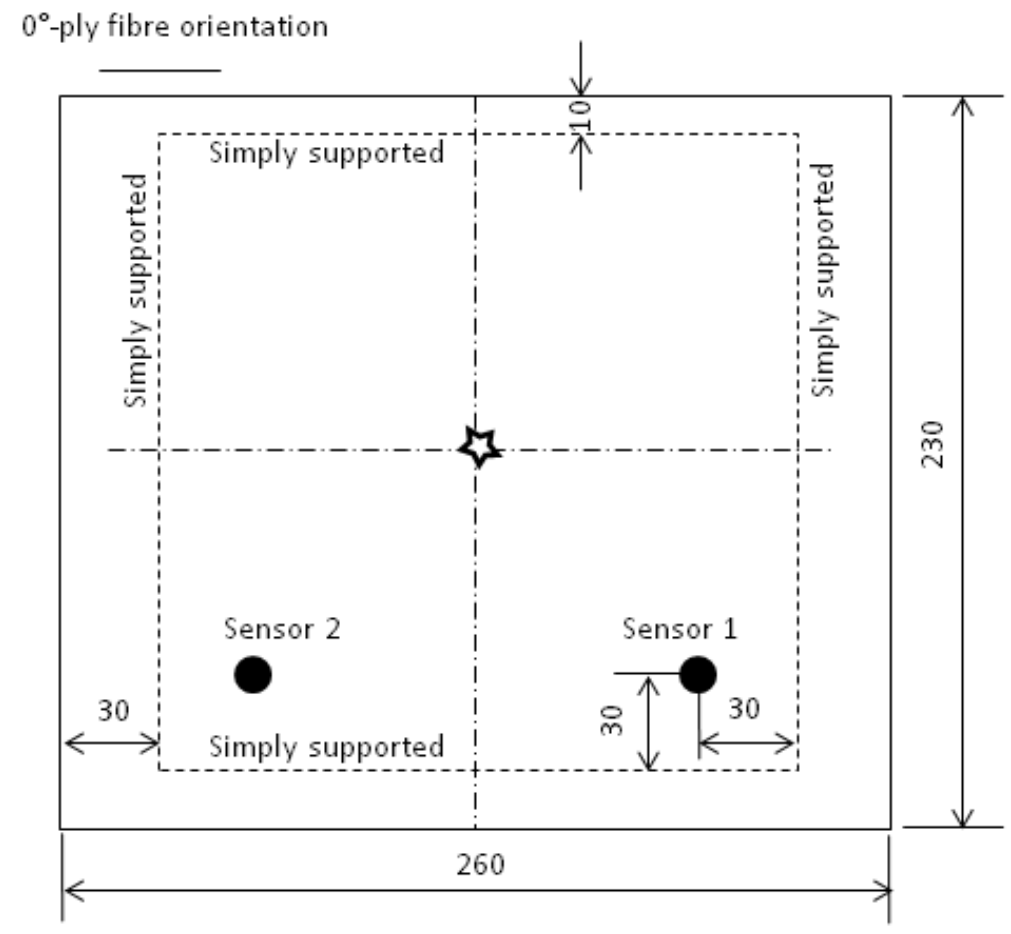

Figure 1 Dimensions of the plate (in $\mathbf{m m}$ ), location of sensors and boundary conditions. 
The experimental and FE results are superimposed in Figure 2. The numerical sensor signals were scaled by the ratio of the peak of the sensor signal measured experimentally to that of the sensor signal predicted numerically, both at sensor 1.There is reasonable agreement between the predicted and experimental impact forces. The FE model predicts slightly higher impact force after $2 \mathrm{~ms}$, which can be a result of high transverse shear stiffness of shell elements. Using more than one continuum shell element (ABAQUS 2010) through the thickness of the plate may improve the predicted force history. Sensor signals contain the first arrived strain wave as well as reflections of the wave from the supports and edges of the plate. Boundary conditions significantly influence the reflected waves. However, perfectly modelling boundary condirions is not trivial. Hence, discrepancies between simulated and experimental signals are expected. Nonetheless, the accuracy of the FE model for simulating impact-induced wave propagation in composite plates is adequate.
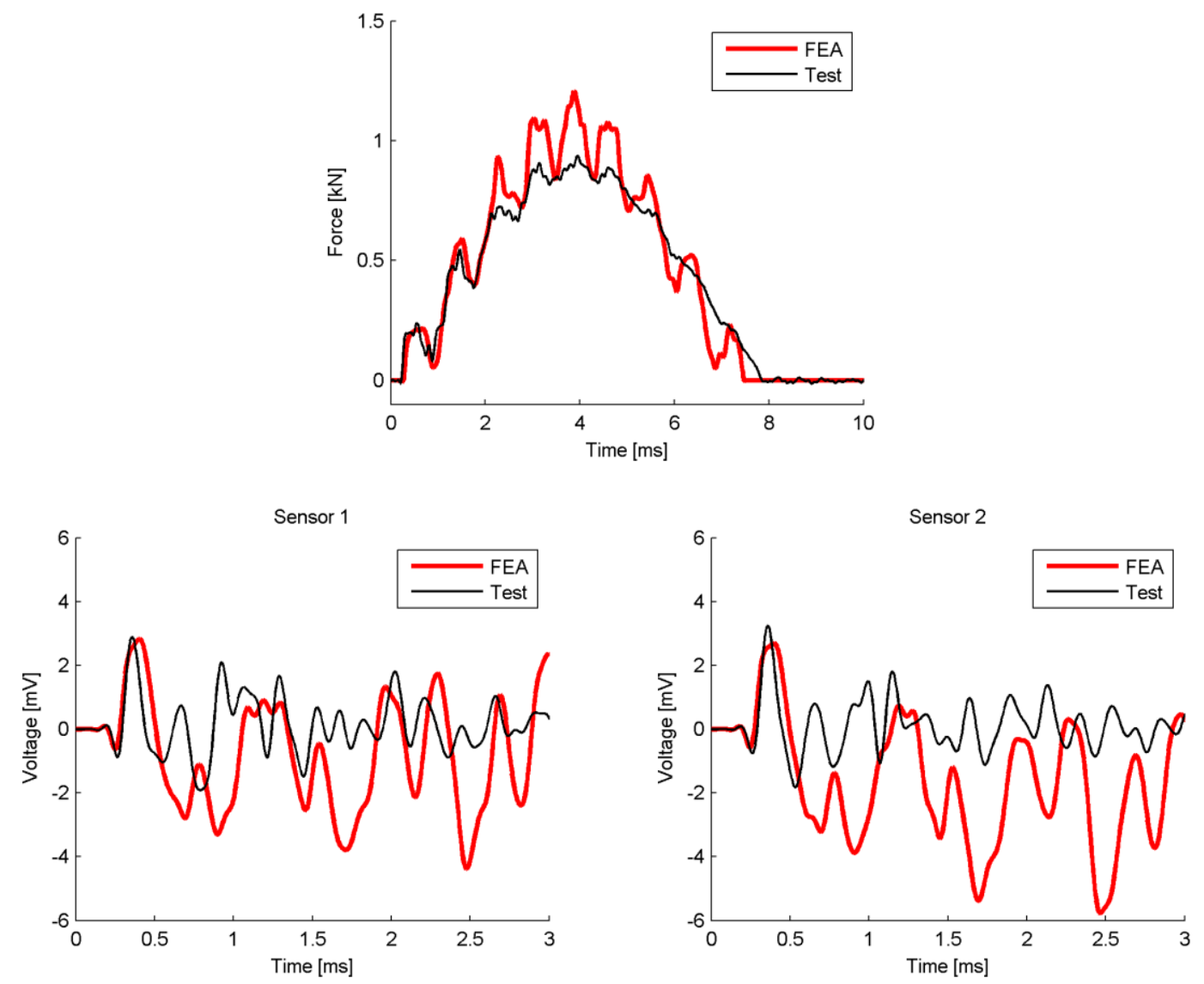

Figure 2 Experimental and FE results for the $2.41 \mathrm{~kg}-0.92 \mathrm{~m} / \mathrm{s}$ impact on the composite plate.

\subsection{Force reconstruction}

The frequency domain deconvolution method was used to reconstruct the impact force history. This method requires spectral components of the system (S). These components were obtained from the steady-state dynamic solution to the numerical model of the plate excited with a harmonic force at the plate centre. Figure 3 shows the magnitude of $\mathrm{S}$ at a range of frequencies. Several peaks in this plot indicate 
reflections of the strain wave from boundaries. Sharp peaks of $S$ would cause a problem if the frequency at which $\mathrm{F}$ is evaluated does not coincide with the frequency at which $S$ is known. In addition, the presence of the boundaries would produce an $S$ that can have zeros. This would potentially cause singularities when evaluating components of $\mathrm{F}$ from eq. (6. However, Martin and Doyle (1996) have shown that the adverse effects of reflections can be alleviated by using the data recorded by two sensors, as follows:

$$
F_{n}=\left[\frac{\bar{S}_{1} U_{1}+\bar{S}_{2} U_{2}}{S_{1}^{2}+S_{2}^{2}}\right]_{n}
$$

The horizontal line indicates complex conjugate.

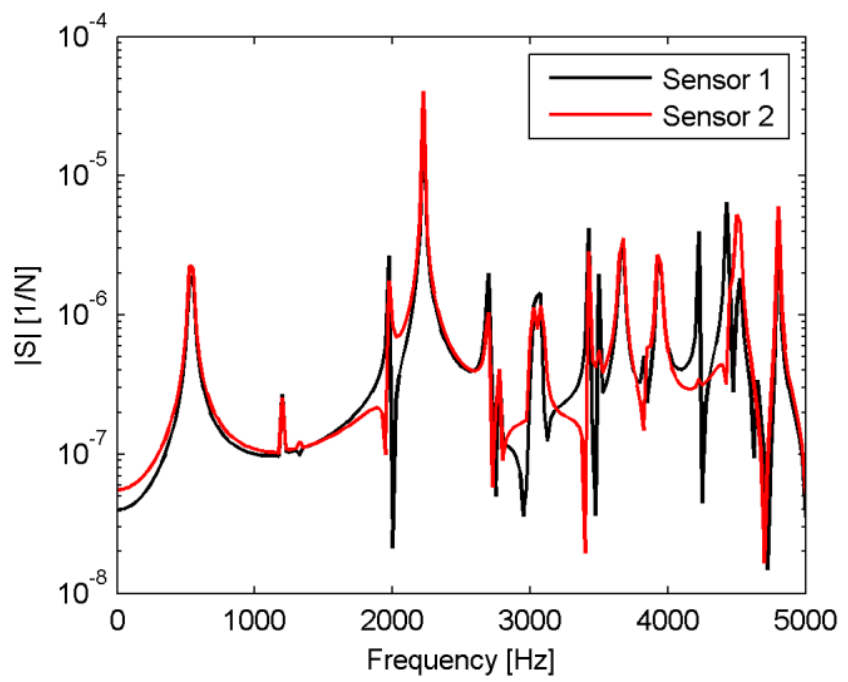

Figure 3 Magnitude of spectral components of the system.

According to Davies and Olsson (2004), when the maximum plate mass affected by impact is smaller than half of the impactor mass, the impact is large mass and the response is quasi-static. In these conditions, significant spectral components of the force and response correspond to frequencies that are lower than the first natural frequency of the system. In the studied problem, the plate mass, between the supports, was approximately $0.18 \mathrm{~kg}$, which is significantly smaller than the impactor mass. According to eq. (5), the frequency of the $12^{\text {th }}$ spectral component, given $20 \mathrm{~ms}$ signal duration, is approximately $550 \mathrm{~Hz}$. This frequency is slightly larger than the first natural frequency of the system, $537 \mathrm{~Hz}$. Therefore, only first twelve spectral components were used in eq. 8 and the rest were set to zero. This also filters the signals from high frequency components and provides a smooth result.

Deflection of thin plates subjected to impacts can be large and, therefore, the nonlinear membrane stiffness of the plate, which affects the contact force, deflection and strain field (Chen et al. 1985), must be considered in numerical solutions. For validation of the FE model, nonlinear stiffening terms were included (nonlinear model). To investigate the influence of these terms on force reconstruction results, another simulation was carried out without including these terms (linear model). Sensor signals were post-processed with the frequency domain deconvolution method and the contact force history was reconstructed. 
The reconstructed and actual forces are plotted in Figure 4. The accuracy of the method is very good for the linear model. For the nonlinear model, however, the reconstructed force is significantly smaller than the actual force. For this model, maximum deflection of the plate was nearly $60 \%$ of its thickness, which indicates that there was moderate geometric nonlinearity in the system.
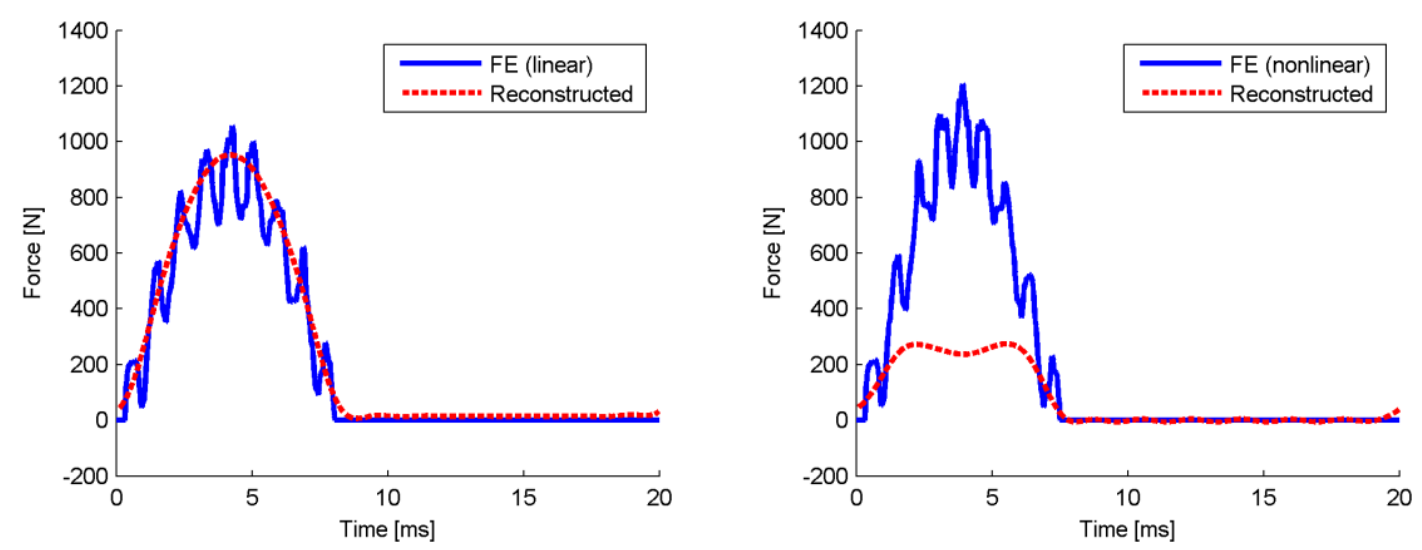

Figure 4 Force reconstruction results for the composite plate.

The delamination threshold force of the plate can be determined using the following relation (Olsson 2001):

$$
F_{d}=\pi \sqrt{16 D^{*} G_{I I C}}
$$

In this equation, $\mathrm{G}_{\text {IIc }}$ is the mode II interlaminar toughness and $\mathrm{D}^{*}$ is the effective plate stiffness approximated by:

$$
D^{*}=\sqrt{D_{11} D_{22}(A+1) / 2} ; \quad A=\left(D_{12}+2 D_{66}\right) / \sqrt{D_{11} D_{22}}
$$

Using $\mathrm{G}_{\text {IIc }}=0.7 \mathrm{~kJ} / \mathrm{m}^{2}$ (Faggiani et al. 2010), the delamination threshold of the laminate is $3700 \mathrm{~N}$. The peak impact force was nearly three-folds smaller than this threshold. Nonetheless, large deflection of the plate drastically influenced the outcome of force reconstruction.

\section{Impact force identification with artificial neural networks}

As it was shown in the previous section, when a panel undergoes large deflection under impact loading, the force reconstruction methods that are based on the convolution integral cannot be used anymore. The generalised form of this integral, which can represent nonlinear time-invariant dynamic systems, is a series of infinite sum of convolutional integrals, called the Volterra series. This series has been used to model nonlinear systems (Tromp et al. 1990; Silva 2005; Balajewicz et al. 2010). Instead of exploring the possibility of using this series for impact identification of nonlinear systems, the neural network technique was used in this study to predict the force history and the peak force. The neural network technique can be used to define complex relationships between inputs and outputs. An artificial neural network is a 
mathematical model which adapts its structure during a learning process. A comprehensive introduction to ANNs can be found in (Bishop 1995; Haykin 2007).

\subsection{Impact data}

To develop and train a neural network capable of identifying impacts of foreign objects on aircraft panels, a large number of sensor data from various impact scenarios, i.e. small mass and large mass impacts, at various locations on the panel are required. However, it is extremely costly and time consuming to obtain these data experimentally. In a previous study (Sharif-Khodaei et al. 2012), a nonlinear FE model of a $2045 \mathrm{~mm}$ by $1070 \mathrm{~mm}$ stiffened panel, made of unidirectional and woven carbon/epoxy composite plies, was developed and used to simulate impacts at various locations. Figure 5 illustrates the impact locations, which were approximately $50 \mathrm{~mm}$ apart. A total of 1265 different impacts, including large mass impacts from the inside of the panel, representing e.g. tool drop, and small mass impacts from the outside of the panel, representing e.g. runway debris impacts, were simulated. Description of the impacts are presented in Table 2.

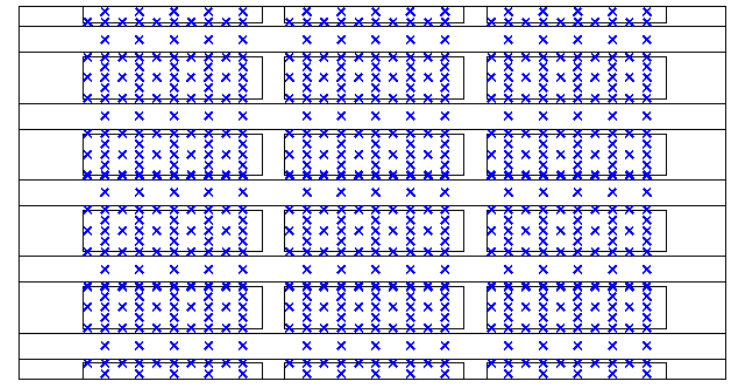

Large mass impacts (from inside)

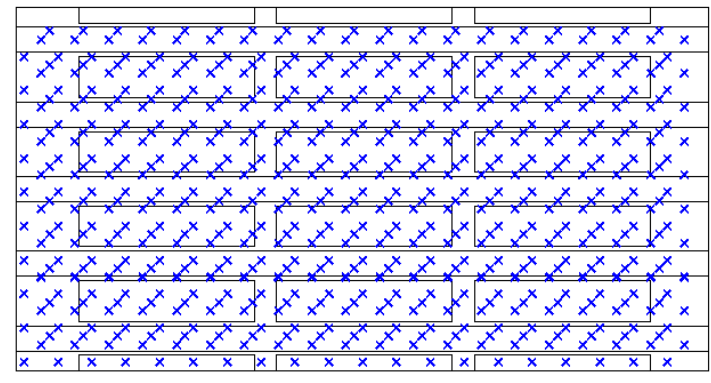

Small mass impacts (from outside)

Figure 5 Location of all simulated impacts.

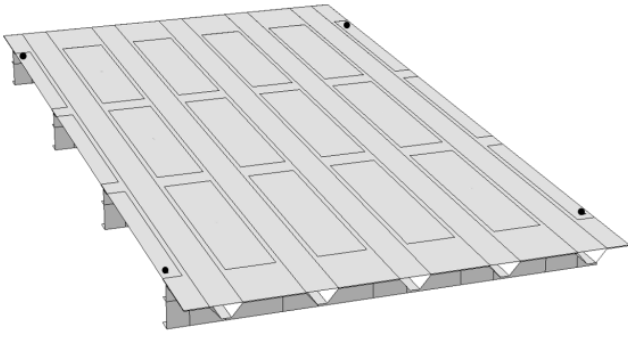

a) 4 sensors in corners of the panel

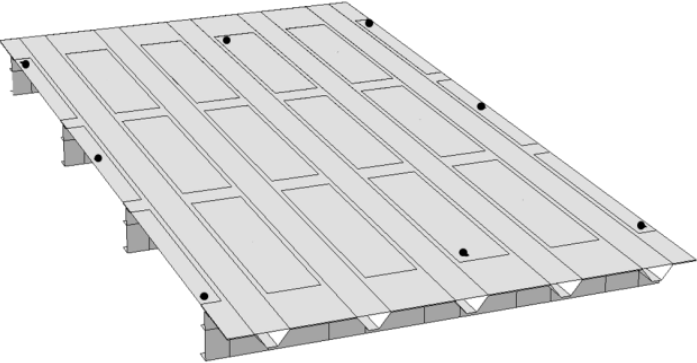

c) 8 sensors

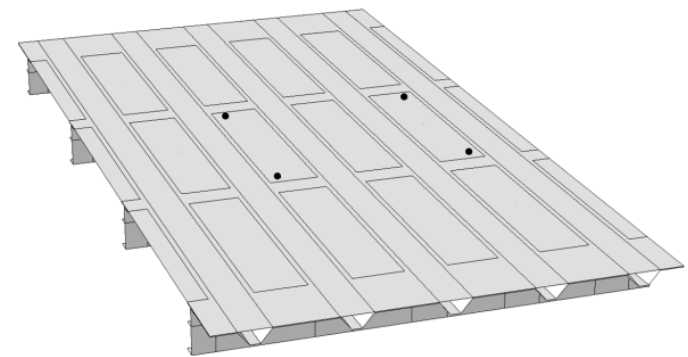

b) 4 sensors in mid-panel

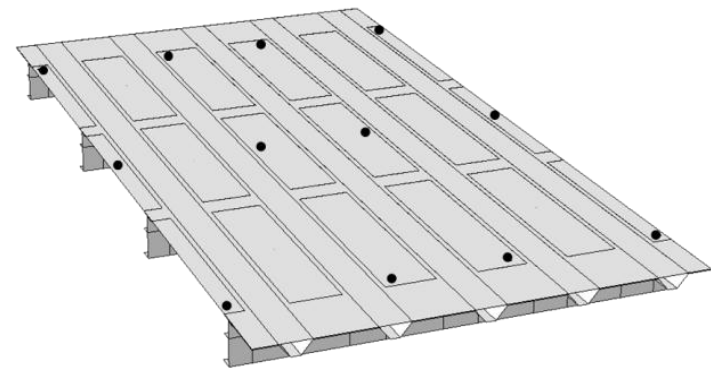

d) 12 sensors

Figure 6 Different sensor distributions. 
During impacts, force history was recorded. In addition, strain data were collected at 300 different sensor positions in order to provide data for sensor optimisation studies, such as (Mallardo et al. 2013). However, in the current study, four different sensor distributions, shown in Figure 6, were used in order to study their influence on the performance of the ANNs.

Table 2 Impact scenarios

\begin{tabular}{c|ccccc}
\hline $\begin{array}{c}\text { Impact } \\
\text { type }\end{array}$ & $\begin{array}{c}\text { Impactor's } \\
\text { diameter } \\
(\mathrm{mm})\end{array}$ & $\begin{array}{c}\text { Impactor's } \\
\text { mass }(\mathrm{kg})\end{array}$ & $\begin{array}{c}\text { Impactor's } \\
\text { velocity }(\mathrm{m} / \mathrm{s})\end{array}$ & $\begin{array}{c}\text { Impactor's } \\
\text { kinetic energy } \\
(\mathrm{J})\end{array}$ & $\begin{array}{c}\text { No. of } \\
\text { impact } \\
\text { scenarios }\end{array}$ \\
\hline Large & 25.4 & 1 & 3 & 4.5 & 225 \\
mass & & 2 & 2 & 4 & 210 \\
& & 3 & 2 & 6 & 210 \\
\hline Small & 12.7 & 0.001 & 25 & 0.31 & 220 \\
mass & & 0.003 & 10 & 0.15 & 200 \\
& & 0.010 & 10 & 0.5 & 200 \\
\hline \multicolumn{5}{r}{} \\
\hline
\end{tabular}

\subsection{Artificial neural network establishment}

Two different networks were established using MATLAB (2011): A) the impact force reconstruction network and B) the peak impact force prediction network. For network A, a few number of spectral components of the sensor data and the same number of spectral components of the impact force were selected to form, respectively, the input layer and the output layer of the ANN. It should be noted that spectral components, except the zero frequency component, are complex numbers (see eq. 4). Hence, each spectral component was represented with its real and imaginary parts in the input layer (see Figure 7). Network A was established for large mass impacts only, because a large number of spectral components of the sensor signal and force should be used to precisely reconstruct the force of small mass impacts, which will render the training process extremely time consuming. For network B, different features were extracted from the sensor signal in order to find the network with the optimum performance.

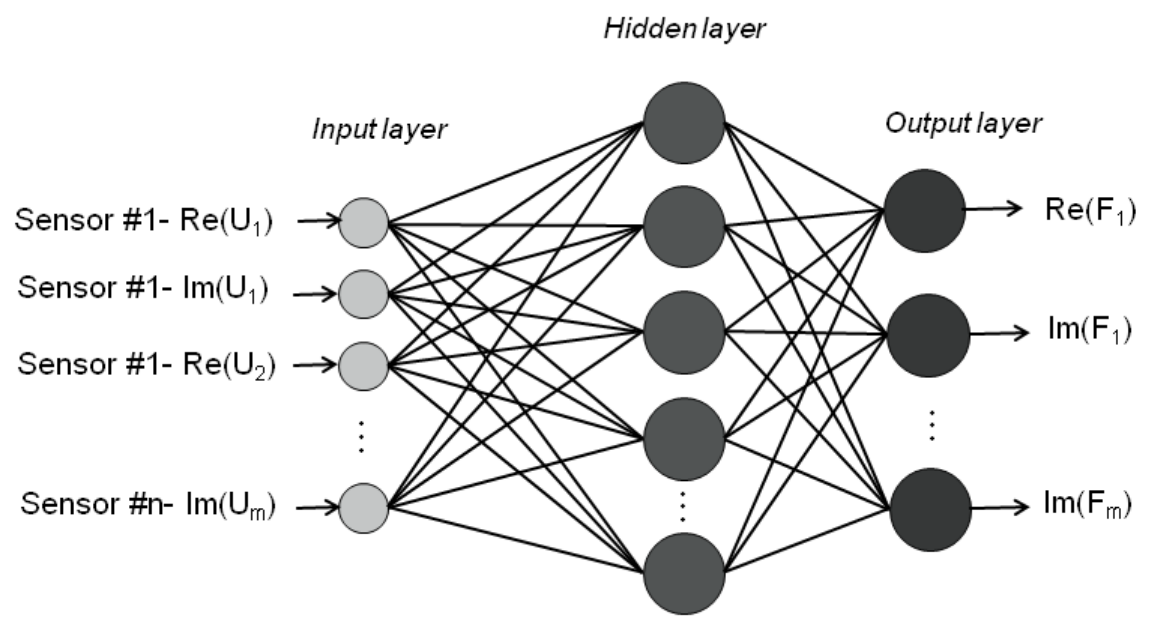

Figure 7 MLP network to reconstruct impact force (network A). 
The ANNs were feedforward Multi-Layer Perceptron (MLP). They were trained with a back propagation learning algorithm. To ensure that the ANNs can make reasonable predictions for new inputs (to generalise the networks), a validation phase was performed on the trained networks (Haykin 2007). Finally, performance of the ANNs was assessed through the test phase. For each phase a different set of impact data was used. The total available data was randomly divided into 50\% training set, $25 \%$ validation set and $25 \%$ test set.

\subsection{Network perfromance}

The training phase of an ANN starts with assigning a set of random weights to the connections, which are repeatedly adjusted according to a learning rule until the error converges to a set limit. Therefore, every time a network is trained, its performance is different. To obtain a robust representation of network performance, each ANN was trained several times (cycles) and its error, on test data, was determined for each cycle. The error function was defined as the normalised mean absolute error between the predicted force and the target force. In (Mallardo et al. 2013), it was shown that the cumulative distribution of the errors does not significantly change after 100 cycles of training. Hence, each network was trained for 100 cycles, and the mean of the error distribution was used to represent the network performance. In addition, the network with the smallest error, among the 100 trained networks, was reported as the best network.

\subsection{Results}

The influence of different signal features, architecture of the network and noise in sensor signals on the performance of ANNs were studied. The study was performed on the composite stiffened panel with 4 sensors in the corners (Figure 6, a). After the features and architecture of the best network was determined, the effect of number and position of sensors was also investigated.

\subsubsection{Signal features}

The inputs to the networks are discrete sensor signals, which contain redundant information about the impact force. Too many input parameters can result in overfitting whereas lack of data would avoid reaching convergance and generalisation. Therefore, often signal pre-processing techniques are used to select/extract appropriate features from the discrete sensor data

For network A, signal features were a few components of the FFT of sensor data. The effect of increasing the number of spectral components from 8 to 12 on the network performance was studied. As can be seen in Table 3, this effect is negligible. Hence, 8 spectral components were selected as an efficient number of input/output parameters per sensor for the rest of this study. Figure 8 shows two examples of simulated (target) and predicted force histories, selected from two different impact scenarios. One impact was in the bay and the other was over the foot of a stringer. The normalised mean absolute error (nmae) between the predicted force and the target force is nearly $18 \%$ for both cases.

The results of using different input parameters for network B are also presented in Table 3. It can be concluded that the case B6 results in the best network performance. For this network, four input parameters are used per sensor: Maximum of the detailed and approximated coefficients of the Discrete Wavelet Transform (DWT) (level 4 Daubechies wavelet) together with the maximum value of the sensor signal and its correspoding time. These signal features were used for the rest of the study. 
Table 3 Influence of different signal features on performance of ANNs

\begin{tabular}{|c|c|c|c|c|}
\hline Case & $\begin{array}{l}\text { Network } \\
\text { architecture }\end{array}$ & Signal features & $\begin{array}{l}\text { Mean error }[\%] \\
\text { (average for } 100 \\
\text { cycles) }\end{array}$ & $\begin{array}{l}\text { Mean error }[\%] \\
\text { (best network out of } \\
100 \text { cycles) }\end{array}$ \\
\hline A1 & [60:20:10:60] & 8 spectral components & 58.95 & 15.78 \\
\hline A2 & {$[76: 20: 10: 76]$} & 10 spectral components & 60.35 & 17.13 \\
\hline A3 & {$[92: 20: 10: 92]$} & 12 spectral components & 57.61 & 15.97 \\
\hline B1 & {$[8: 20: 10: 1]$} & Max signal + corresponding time & 35.34 & 28.04 \\
\hline B2 & {$[4: 20: 10: 1]$} & Max signal & 40.45 & 23.19 \\
\hline B3 & {$[8: 20: 10: 1]$} & Max filtered signal $*+\max$ signal & 40.92 & 21.13 \\
\hline B4 & {$[8: 20: 10: 1]$} & $\begin{array}{l}\text { Max approx. coefficient of DWT } \\
\text { transform + max signal }\end{array}$ & 33.45 & 22.16 \\
\hline B5 & {$[12: 20: 10: 1]$} & $\begin{array}{l}\text { Max approx. + detailed coeff. of } \\
\text { DWT transform + max signal }\end{array}$ & 27.56 & 19.4 \\
\hline B6 & {$[16: 20: 10: 1]$} & $\begin{array}{l}\text { Max approx. + detailed coeff. of } \\
\text { DWT transform + max signal + } \\
\text { time corresponding to max signal }\end{array}$ & 26.15 & 18.22 \\
\hline
\end{tabular}

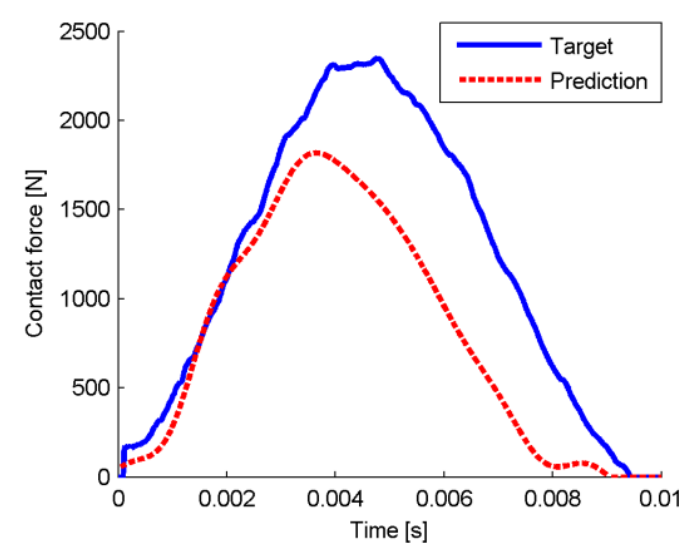

(a)

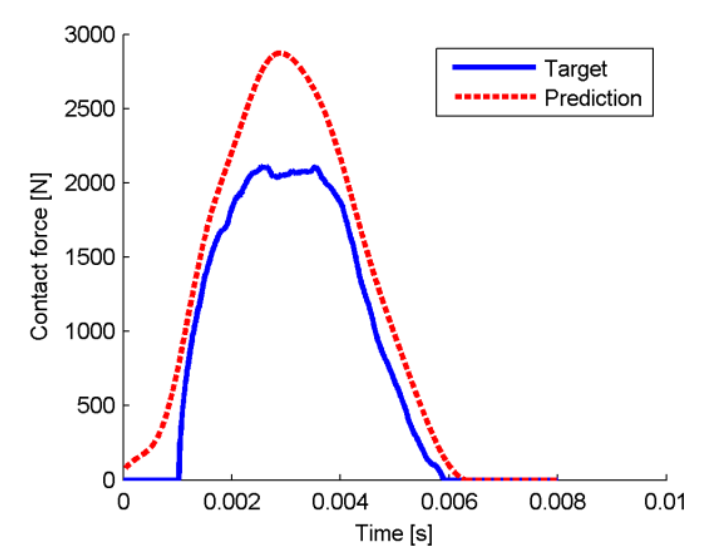

(b)

Figure 8 Examples of force history prediction with network A, a) $3 \mathrm{~kg}-2 \mathrm{~m} / \mathrm{s}$ impact in the bay, $n m a e=18.5 \%$ and b) $2 \mathrm{~kg}-2 \mathrm{~m} / \mathrm{s}$ impact over the stringer foot, $\mathrm{nmae}=17.9 \%$.

\subsubsection{Network Architecture}

Architecture of an ANN, e.g. the number of hidden layers, number of neurons per layer and type of activation functions, infuences its training time and performance. Unfortunately, there are no set rules to find the optimum network architecture for a given problem. Therefore, a parametric study was carried out to find the influence of the architecture of the ANNs on their performance.

The number of hidden layers and neurons per layer were varied to test the network performance for each case. The results are presented in Table 4. For network A, the best performance was associated with a network with one hidden layer of 10 neurons (case A6). For network B, the best performance was obtained with two hidden layers of 10 and 5 neurons, respectively (case B7).

The training algorithm used so far was Levenberg-Marquardt (LM) backpropagation. Other algorithms were also used to train the networks, namely:

- GDA: Gradient descent backpropagation

- SCG: Scaled conjugate gradient backpropagation 
- BR: Bayesian regulation backpropagation

- CGF: Conjugate gradient backpropagation with Fletcher-Reeves updates

As it can be seen in Table 4, for network A trained with the SCG and GDA algorithms (cases A7 and A9), the mean error for 100 training cycles was significantly smaller than the mean error of the other cases. In addition, this error was similar to the mean error of the best network. This implies that SCG and GDA algorithms result in consistent networks with respect to the mean error. For network B, the LM learning algorithm provided the best perfromance (case B7), which agrees with the findings of (Lyn Dee et al. 2011).

Table 4 Influence of different network architecture on performance of ANN

\begin{tabular}{c|llll}
\hline Case & Layers & Training algorithm & $\begin{array}{l}\text { Mean error [\%] } \\
\text { (average for 100 } \\
\text { cycles) }\end{array}$ & $\begin{array}{l}\text { Mean error [\%] } \\
\text { (best network out } \\
\text { of 100 cycles) }\end{array}$ \\
\hline A4 & {$[60: 10: 5: 60]$} & LM & 50.38 & 16.36 \\
A5 & {$[60: 20: 60]$} & LM & 64.32 & 13.66 \\
A6 & {$[\mathbf{6 0 : 1 0 : 6 0 ]}$} & LM & $\mathbf{5 1 . 3 6}$ & $\mathbf{1 3 . 0 3}$ \\
\hline A7 & {$[60: 10: 60]$} & CGF & 19.44 & 17.09 \\
A8 & {$[60: 10: 60]$} & BR & Did not converge & \\
A9 & {$[\mathbf{6 0 : 1 0 : 6 0 ]}$} & SCG & $\mathbf{1 8 . 9 6}$ & $\mathbf{1 5 . 6 1}$ \\
A10 & {$[60: 10: 60]$} & GDA & 66.58 & 29.47 \\
\hline B7 & {$[\mathbf{1 6 : 1 0 : 5 : 1 ]}$} & LM & $\mathbf{2 5 . 8 9}$ & $\mathbf{1 8 . 2 8}$ \\
B8 & {$[16: 20: 1]$} & LM & 31.22 & 21.86 \\
B9 & {$[16: 10: 1]$} & LM & 30.7 & 22.45 \\
\hline B10 & {$[16: 10: 5: 1]$} & CGF & 34.37 & 26.91 \\
B11 & {$[16: 10: 5: 1]$} & BR & 114.42 & 114.39 \\
B12 & {$[16: 10: 5: 1]$} & SCG & 36.18 & 27.09 \\
B13 & {$[16: 10: 5: 1]$} & GDA & 49.44 & 35.86 \\
\hline
\end{tabular}

\subsubsection{Influence of noise}

Under real load conditions, there may be considerable levels of noise in sensor data due to the environmental and technical factors. Therefore, to produce sensor signals comparable to life-time scenarios, white Gaussian noise was added to the data. The amount of added noise was $5 \%$ of the maximum sensor signal (a signal-to-noise ratio, SNR, of $26 \mathrm{~dB}$ ). Network A9 (Table 4) was trained for 100 cycles using the noisy data. The mean error was evaluated as $18.76 \%$, which is almost equal to the mean error of network A9. This indicates that the effect of the added noise on the predictions of the network is negligible. This can be explained by recalling the fact that only first 8 spectral components of the sensor signal were extracted and used in the input layer, which effectively filtered the signal from large frequency components, including noise.

Some of the input features to network B were obtained from the Wavelet transform of the sensor signal, which has a filtering effect. The Wavelet transform is constituted by different levels. One factor that affects the selection of the number of levels is the SNR. Moreover, the mother wavelet must be carefully chosen to better approximate and capture the transient spikes of the original signal. Therefore, the level of decomposition and the choice of mother wavelet function on the perfromance of network B, established with noisy data, were studied. The type of wavelet functions which were considered are Daubechies (db), Haar, Biorthogonal (bior), Symlets 
(sym), Coiflets (coif) and Discerete Meyer (dmey). The input parameters used for estabishing the ANN were the same as case B6 (Table 3). The results showed that presence of noise did not significantly change the performance of the network because the signals were processed with the DWT. In addition, different types of mother wavelet signals and decomposition levels had marginal influence on the training of the networks. The mother wavelet function which resulted in the best network performance was the Haar wavelet. This mother wavelet is the simplest wavelet function and represnets the same wavelet as Daubechies level 1.

\subsection{Categorization of impact scenarios}

It has been demonstrated (Olsson 2000) that the impact response of plates is governed by the impactor-plate mass ratio, which leads to the categorisation of impacts to small mass and large mass. Small mass impacts have short durations and cause a flextural wave controlled response in the plate, whereas large mass impacts have long durations and cause a quasi-static response in the plate. This leads to very different contact forces and sensor signals during large mass and small mass impacts. The contact force and structural response of large mass impacts were represented with a few components of their frequency domain spectrum, but for small mass impacts many more spectral components were needed.

Network A was trained for large mass impacts. However, network B was trained with both types of impacts. The performance of this network was improved by establishing ANNs for small mass impacts and large mass impacts, as can be seen in Table 5. The error of the large mass network (case B14) is less than one-half the error of the small mass network (case B15) or the combined network (case B7). This can be attributed to different impact responses. The question which remains is how to identify the type of impacts by using the sensor data.

Table 5 ANNs trained with different impact data

\begin{tabular}{l|llll}
\hline Case & Layers & Impact data & $\begin{array}{l}\text { Mean error [\%] } \\
\text { (average for 100 } \\
\text { cycles) }\end{array}$ & $\begin{array}{l}\text { Mean error [\%] } \\
\text { (best network out of } \\
100 \text { cycles) }\end{array}$ \\
\hline B14 & {$[16: 10: 5: 1]$} & Large mass impacts - with noise & 12.34 & 9.98 \\
B15 & {$[16: 10: 5: 1]$} & Small mass impacts - with noise & 28.03 & 20.76 \\
\hline
\end{tabular}

The quasi-static and wave controlled responses of a plate subjected to respectively large mass and small mass impacts suggest that spectral components of the strain data (structural response) also contain different energy distributions. In large impacts, the spectral sensor data probably contain large energies at lower frequencies. To examine if there is any distinction between dominant spectral components (in terms of energy) of sensor data recorded during large mass and small mass impacts, the dominant spectral component of each sensor signal was determined for each impact. Then, the spectral component with the smallest frequency was selected to represent the dominant spectral component of the impact. In total, 1265 dominant components were determined and their corresponding frequency was plotted in a histogram, which is presented in Figure 9 for four corner sensors (Figure 6, a). It can be observed that the small mass impacts have a very scattered dominant frequency, while the large mass impacts have a more unified behaviour. This may further explain why the error of the ANN trained for small mass impacts was much higher than the error of the ANN 
trained for large mass impacts. The histogram also reveals a distinct threshold frequency between the two types of impacts. Similar histograms were obtained for the other sensor distributions shown in Figure 6.

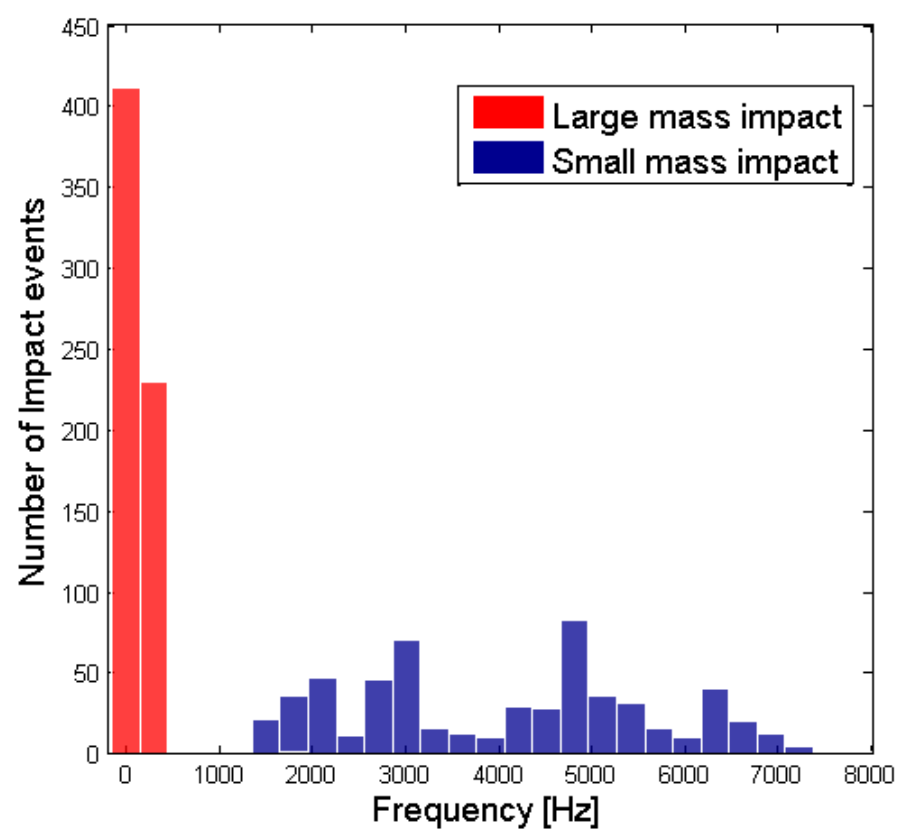

Figure 9 Dominant frequencies of impacts - four corner sensors.

\subsection{Influence of sensor topology on error distribution}

The number and position of sensors may greatly influence the performance of the ANNs. To find the best sensor distribution, an exhaustive search or an optimisation analysis should be carried out (Staszewski et al. 2000; Mallardo et al. 2013). In this study, however, four different sensor distributions, shown in Figure 6, were considered. As it was shown in section 4.5, large mass impacts have more unified behavior, which results in smaller error for the trained network. Therefore, the influence of sensor typology on impact identification was studied using the large mass impact network. The network architectures were the same as those of cases A9 and B14 presented in Table 4 and Table 5, respectively. The mean error of each network is presented in Table 6 . This table indicates that as a result of increasing the number of sensors, the mean error slightly decreases.

Table 6 Influence of sensor topology on performance of ANNs

\begin{tabular}{l|llll}
\hline Case & Layers & Sensor distribution & $\begin{array}{l}\text { Mean error [\%] } \\
\text { (average for 100 } \\
\text { cycles) }\end{array}$ & $\begin{array}{l}\text { Mean error [\%] } \\
\text { (best network out of } \\
\text { 100 cycles) }\end{array}$ \\
\hline A11 & {$[60: 10: 60]$} & 4 sensors in mid-panel & 16.33 & 13.45 \\
A12 & {$[120: 10: 120]$} & 8 sensors & 15.63 & 13.71 \\
A13 & {$[\mathbf{1 8 0 : 1 0 : 1 8 0 ]}$} & 12 sensors & $\mathbf{1 5 . 3 4}$ & $\mathbf{1 3 . 4 6}$ \\
\hline B16 & {$[16: 10: 5: 1]$} & 4 sensors in mid-panel & 15.69 & 12.09 \\
B17 & {$[32: 10: 5: 1]$} & 8 sensors & 12.05 & 8.73 \\
B18 & {$[\mathbf{4 8 : 1 0 : 5 : 1 ]}$} & 12 sensors & $\mathbf{1 1 . 9 4}$ & $\mathbf{8 . 5 4}$ \\
\hline
\end{tabular}




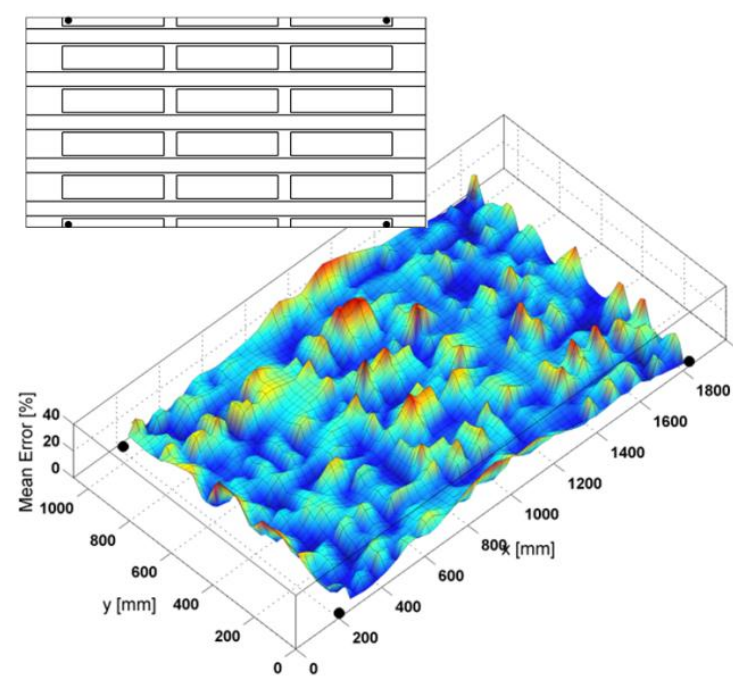

a) 4 sensors in corners

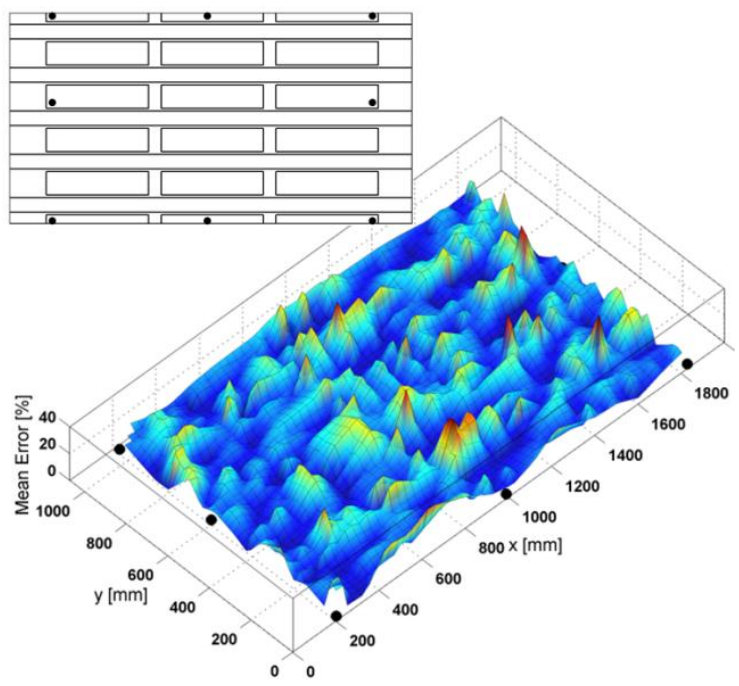

c) 8 sensors

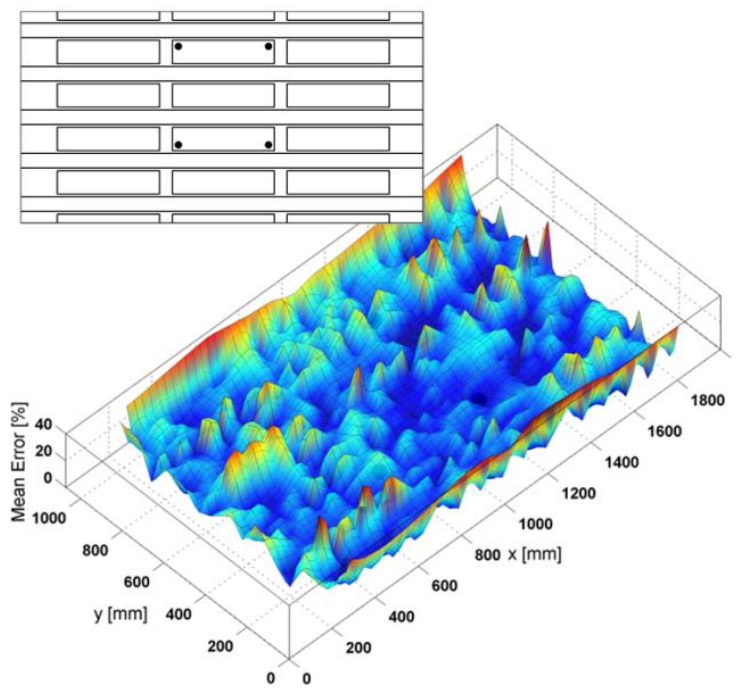

b) 4 sensors in mid-panel

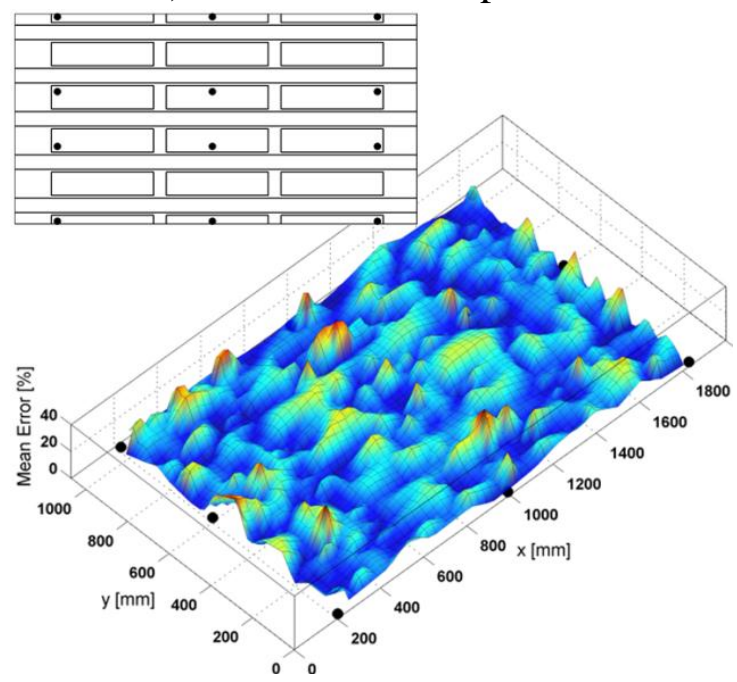

d) 12 sensors

Figure 10 Error distribution for different sensor topologies.

In Figure 10, performance of network B is presented as a surface plot of the mean error on the testing data at corresponding impact locations. Comparing cases (a) and (b), it can be seen that when sensors are placed close to the centre of the panel, estimations for impacts which occur outside of the sensor network, i.e. close to edges, have higher errors than when sensors are placed in corners of the plate, thus surrounding all impacts. When sensors are in corners of the panel, some peak errors can be identified near the middle of the panel. Most of these peaks were removed by adding more sensors to the ANNs, i.e. cases (c) and (d).

Another way of representing the results of the trained networks is by plotting the Cumulative Distribution Function (CDF), which presents the probability of detection of the trained network against the mean error. CDF is obtained by integrating the Probability Density Function (PDF). To obtain the PDF for each trained ANN, a large number of data is needed. This is impossible to obtain experimentally and the computational cost is extremely high. Therefore, to generate new sets of impact data, which have not been used in the training phase, Gaussian noise was added to the full range of simulated sensor data (at each discrete value). Altogether, for each trained 
network configuration shown in Figure 6, 123,500 input data were generated. The CDFs evaluated for network B (peak force prediction) are shown in Figure 11. As can be seen, the best trained network is the one with 12 sensors. It is worth noting that the mean error of the case with 8 corner sensors is similar to that of the case with 4 corner sensors, suggesting that solely increasing the number of sensors does not necessarily improve the performance of ANNs.

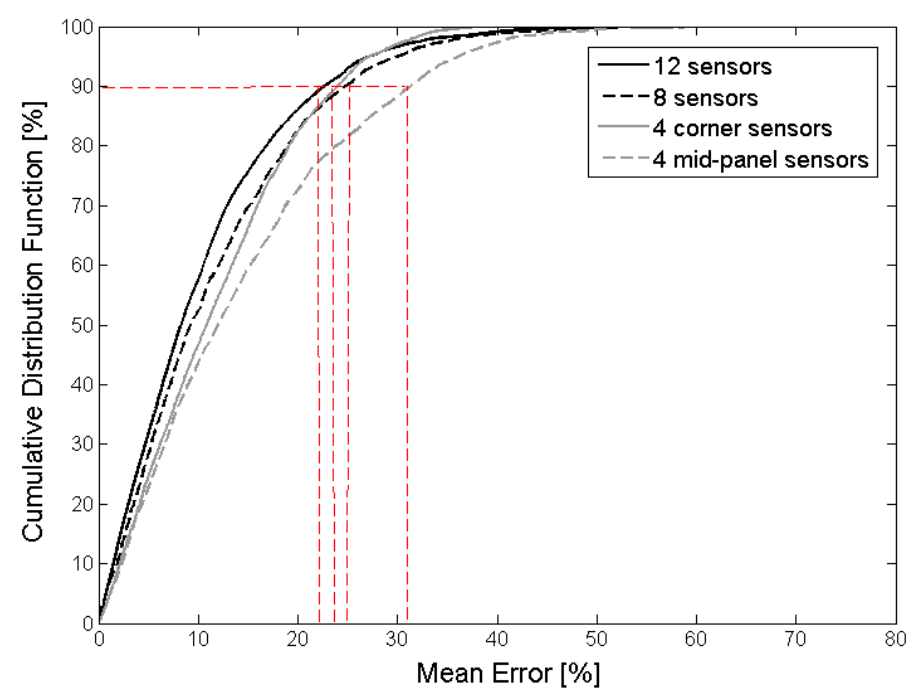

Figure 11 Probabilistic behaviour of the error function for different trained ANNs network $B$.

\section{Discussion}

Accurate and reliable identification of the impact force would eventually result in lower design, production and maintenance costs of aircraft structures. Several methods have been suggested for indentifying the impact force (Doyle 1987; Inoue et al. 1991; Martin et al. 1996; Doyle 1997; Park et al. 2009), which are all based on the convolution relation. This relation is valid for linear dynamic systems, such as thinwalled structures which undergo small deflection under loading. However, it was shown in this paper that a composite plate, representing a typical bay of aircraft panels, can undergo large deflection under an impact with a load level that is well below the delamination threshold of the plate. This agrees with the results of (Chen et al. 1985; Abrate 2001). As a result, the frequency domain deconvolution method failed to provide a reasonable prediction of the impact force history. These findings suggest that convolution-based methods are not applicable to force identification of aircraft panels subjected to threatening impacts of foreign objects.

It should be noted that the focus of this study was on geometric nonlinearity due to large deflection. Nonlinearities related to damage growth have not been considered. Impact force identification in presence of structural damage will be the subject of a future study.

The artificial neural network technique, which is able to establish nonlinear relationships between inputs and outputs, was used to reconstruct the impact force and estimate the peak impact force for in-service impacts, including large mass and small mass impacts. Estimation of the contact force with ANNs has been reported in (Chandrashekhara et al. 1998), where a nonlinear FE model of a composite plate 
$(127 \times 127 \mathrm{~mm} 2)$ was developed and used to generate strain-force data for training and testing the network. Only small mass impacts at one location were simulated. Furthermore, the aim was to estimate the impact force at some time intervals of one force history by using the force data of the same force history at other time intervals. Hence, the proposed networks are probably not able to predict the force history of a new impact. In the current study, however, the ANNs were established for a composite stiffened panel $\left(2045 \times 1070 \mathrm{~mm}^{2}\right)$ subjected to different types of impacts. Furthermore, the networks did not need the impact location a priori. The performance of the force reconstruction network (network A) and peak force prediction network (network B) can probably be improved by using data from impacts at the same location but with a different force magnitude. In addition, since spectral components of the force and sensor signals are complex numbers, complex-valued ANNs may provide better results for force reconstruction (network A). Future work may focus on developing a code for establishing such networks and applying them to the impact force reconstruction problem.

The best trained network for estimating peak impact force resulted in $17 \%$ mean error on the test set. This is a significant improvement as compared with $26 \%$ and $28 \%$ mean error reported by (Ghajari et al. 2012) and (Staszewski et al. 2000), respectively. In (Ghajari et al. 2012), both small mass and larges mass impacts were considered, but in (Staszewski et al. 2000), the impact force was extemely low (maximum $0.1 \mathrm{~N}$ ). By establishig a separate network for large mass impacts, the mean error was decreased to less than $10 \%$. The mean error, however, remained high (approximately 20\%) for the ANN established for small mass impacts. Furthur work needs to be carried out to improve this network.

It was shown that the number and position of sensors affect the prediction error. The error was decreased when sensors surounded the impact area. In addition, although increasing the number of sensors decreased the error, its effect was not as much as the effect of sensor position. The availability of data for a large number of sensors and the simple structure of the ANNs, which reduces the training time, makes the proposed FE-based methodology suitable for future studies on optimisation of sensor distribution.

\section{Conclusions}

It is shown that methods based on the convolution integral (linearity assumption) produce accurate results for impacts that cause small deflections in composite panels. However, they fail to provide reasonable results for moderate impacts, which occur during manufacturing or operation, because these impacts cause large deflections. ANNs have been etablished to identify the force of large mass and small mass impacts on a composite stiffened panel. A parametric study has been performed to reveal the influence of signal features, network architecture, noise and sensor distribution on the performance of the ANNs. Even though the panel undergoes large deflections under impacts, the networks have been able to predict the peak impact force with acceptable accuracy. Categorising impacts into large mass and small mass, and training separate networks for each of them has improved the predictions. Frequency of the dominant spectral component (with respect to energy) of the sensor signal appears to be a suitable parameter to distinguish between large mass and small mass impacts. 


\section{Acknowledgment}

This work was partially funded by the SMASH project as part of the CleanSky, JTICS-2009-01-GRA-01-005, research programme.

\section{References}

ABAQUS (2010). Abaqus Analysis User's Manual, Simulia.

Abrate, S. (2001). "Modeling of impacts on composite structures." Composite Structures 51(2): 129-138.

Adams, R. and J. F. Doyle (2002). "Multiple force identification for complex structures." Experimental Mechanics 42(1): 25-36.

Balajewicz, M., F. Nitzsche and D. Feszty (2010). "Application of multi-input volterra theory to nonlinear multi-degree-of-freedom aerodynamic systems." AIAA journal 48(1): 56-62.

Bishop, C. M. (1995). Neural networks for pattern recognition, Oxford university press.

Chandrashekhara, K., A. C. Okafor and Y. P. Jiang (1998). "Estimation of contact force on composite plates using impact-induced strain and neural networks." Composites Part B: Engineering 29(4): 363-370.

Chen, J. and C. Sun (1985). "Dynamic large deflection response of composite laminates subjected to impact." Composite Structures 4(1): 59-73.

Dae-Un, S., O. Jung-Hoon, K. Chun-Gon and H. Chang-Sun (2000). "Impact monitoring of smart composite laminates using neural network and wavelet analysis." Journal of intelligent material systems and structures 11(3): 180190.

Davies, G. A. O. and R. Olsson (2004). "Impact on composite structures." The Aeronautical Journal: 541-563.

Doyle, J. (1987). "Experimentally determining the contact force during the transverse impact of an orthotropic plate." Journal of sound and vibration 118(3): 441448.

Doyle, J. F. (1997). "A wavelet deconvolution method for impact force identification." Experimental Mechanics 37(4): 403-408.

Faggiani, A. and B. Falzon (2010). "Predicting low-velocity impact damage on a stiffened composite panel." Composites Part A: Applied Science and Manufacturing 41(6): 737-749.

Ghajari, M., Z. Sharif Khodaei and M. Aliabadi (2012). "Impact Detection Using Artificial Neural Networks." Key Engineering Materials 488: 767-770.

Gopalakrishnan, S. and J. Doyle (1994). "Wave propagation in connected waveguides of varying cross-section." Journal of sound and vibration 175(3): 347-363.

Haykin, S. S. (2007). Neural networks: a comprehensive foundation, Prentice Hall Englewood Cliffs, NJ.

Haywood, J., P. T. Coverley, W. J. Staszewski and K. Worden (2005). "An automatic impact monitor for a composite panel employing smart sensor technology." Smart Materials and Structures 14: 265.

Inoue, H., J. J. Harrigan and S. R. Reid (2001). "Review of inverse analysis for indirect measurement of impact force." Applied Mechanics Reviews 54: 503.

Inoue, H., H. Ishida, K. Kishimoto and T. Shibuya (1991). "Measurement of impact load by using an inverse analysis technique: Comparison of methods for estimating the transfer function and its application to the instrumented charpy 
impact test." JSME international journal. Ser. 1, Solid mechanics, strength of materials 34(4): 453-458.

Jankowski, Ł. (2009). "Off-line identification of dynamic loads." $\underline{\text { Structural and }}$ Multidisciplinary Optimization 37(6): 609-623.

Jones, R. T., J. S. Sirkis and E. Friebele (1997). "Detection of impact location and magnitude for isotropic plates using neural networks." Journal of intelligent material systems and structures 8(1): 90-99.

Lyn Dee, G., N. Bakhary, A. Abdul Rahman and B. Hisham Ahmad (2011). "A Comparison of Artificial Neural Network Learning Algorithms for VibrationBased Damage Detection." Advanced Materials Research 163: 2756-2760.

Mallardo, V., M. Aliabadi and Z. S. Khodaei (2013). "Optimal sensor positioning for impact localization in smart composite panels." Journal of intelligent material systems and structures 24(5): 559-573.

Martin, M. T. and J. F. Doyle (1996). "Impact force identification from wave propagation responses." International Journal of Impact Engineering 18(1): $65-77$.

MATLAB (2011). The Mathworks Inc.

Olsson, R. (2000). "Mass criterion for wave controlled impact response of composite plates." Composites Part A: Applied Science and Manufacturing 31(8): 879887.

Olsson, R. (2001). "Analytical prediction of large mass impact damage in composite laminates." Composites Part A: Applied Science and Manufacturing 32(9): 1207-1215.

Park, J., S. Ha and F. K. Chang (2009). "Monitoring Impact Events Using a SystemIdentification Method." AIAA journal 47: 2011-2020.

Pierson, M. O. and R. Vaziri (1996). "Analytical solution for low-velocity impact response of composite plates." AIAA journal 34(8): 1633-1640.

Sekuła, K., C. Graczykowski and J. Holnicki-Szulc (2013). "On-line impact load identification." Shock and Vibration 20(1): 123-141.

Sharif-Khodaei, Z., M. Ghajari and M. Aliabadi (2012). "Determination of impact location on composite stiffened panels." Smart Materials and Structures 21(10): 105026.

Silva, W. (2005). "Identification of nonlinear aeroelastic systems based on the Volterra theory: progress and opportunities." Nonlinear Dynamics 39(1-2): 2562.

Staszewski, W. J., K. Worden, R. Wardle and G. R. Tomlinson (2000). "Fail-safe sensor distributions for impact detection in composite materials." $\underline{\text { Smart }}$ Materials and Structures 9: 298.

Tromp, J. and J. Jenkins (1990). "A Volterra kernel identification scheme applied to aerodynamic reactions." AIAA Paper(90-2803): 20-22.

Worden, K. and W. J. Staszewski (2000). "Impact location and quantification on a composite panel using neural networks and a genetic algorithm." Strain 36(2): 61-68. 